\title{
Du populisme bureaucratique dans l'histoire institutionnelle du développement rural en Afrique de l'ouest
}

Jean-Pierre Chauveau

\section{(2) OpenEdition Journals}

Édition électronique

URL : http://journals.openedition.org/apad/3763

DOI : 10.4000/apad.3763

ISSN : 1950-6929

Éditeur

LIT Verlag

\section{Édition imprimée}

Date de publication : 1 décembre 1992

\section{Référence électronique}

Jean-Pierre Chauveau, « Du populisme bureaucratique dans l'histoire institutionnelle du développement rural en Afrique de l'ouest », Bulletin de l'APAD [En ligne], 4 | 1992, mis en ligne le 26 juin 2008, consulté le 08 septembre 2020. URL : http://journals.openedition.org/apad/3763 ; DOI : https:// doi.org/10.4000/apad.3763

Ce document a été généré automatiquement le 8 septembre 2020.

Bulletin de I'APAD 


\section{Du populisme bureaucratique dans l'histoire institutionnelle du développement rural en Afrique de l'ouest}

Jean-Pierre Chauveau

1 Dans le précédent Bulletin de l'APAD, j'avais essayé de montrer, à partir du matériel historiographique, que, loin d'être "alternative", la conception contemporaine du "développement participatif" ou "à partir de la base" s'inscrit dans le modèle de référence élaboré à partir de la Première Guerre mondiale par les experts coloniaux. L'explication de cette récurrence ne se satisfaisant pas de l'habituelle opposition entre un discours qui serait purement idéologique et des pratiques qui seraient dictées par le seul intérêt, nous avions avancé une autre interprétation qui, sans exclure la première, prend en compte le système de valeurs caractéristique de la "culture du développement" telle que la perçoivent et la mettent en action les opérateurs de développement. Il s'agit, par conséquent, d'élaborer une anthropologie culturelle, voire cognitive, des agents du développement (Chauveau, 1992).

Le dispositif de Développement désigne ainsi non seulement les structures concrètes et organisées de conception et d'administration des interventions (incluant les agents spécialisés et hiérarchisés qui la mettent en œuvre: responsables politiques et administratifs, experts, personnel local...) mais aussi un ensemble (complexe, nous le verrons...) de représentations et de normes acquises structurant les manières de percevoir, de penser et d'agir des acteurs. Un tel dispositif constitue une véritable institution sociale dans laquelle la culture "commune" du développement est redoublée par une culture d'organisation ou professionnelle, avec ses variantes correspondant aux positions sociales des différentes catégories d'agents au sein de leurs organisations et vis-à-vis de la société globale, ou correspondant aux contextes spécifiques (historiques, nationaux et internationaux) de ce dispositif. La professionnalisation du développement contribue paradoxalement à renforcer encore les dispositions mentales 
inconscientes des acteurs sociaux relativement aux valeurs que la culture occidentale associe à la notion de développement. C'est donc à rendre compte de ces dispositions largement inconscientes (ce qui est précisément le propre des "habitus" culturels) que nous allons nous attacher en développant les lignes directrices annoncées dans notre précédente contribution en priant le lecteur de nous excuser de quelques répétitions inévitables.

3 Nous reprendrons d'abord les deux composantes fondamentales du modèle participatif de développement: a) l'organisation bureaucratique comme idéal-type d'autorité légitime, et b) le populisme comme conception idéale-typique que se font les développeurs de leurs rapports aux populations paysannes africaines telles qu'ils se les représentent (la notion d'idéal-type, tirée de Max Weber, désigne le concept abstrait réunissant un ensemble de traits caractéristiques d'un phénomène historiquement observé).

4 Nous décrirons ensuite comment la contradiction permanente de ces deux composantes aboutit paradoxalement à la reproduction du populisme bureaucratique comme idéal-type, à peu près inchangé depuis le début de la colonisation, de la culture du développement. Nous déclinerons enfin, à partir du modèle central, les sources de variabilité qui peuvent rendre compte de ses variantes dans l'histoire institutionnelle du développement rural et qui aboutissent, en fin de compte, à réactualiser sans cesse le modèle populiste-bureaucratique dominant.

Les composantes "bureaucratique" et "populiste" de la culture des développeurs

Limitons-nous provisoirement aux catégories d'agents prééminents qui pourvoient l'institution de Développement en systèmes de normes, c'est-à-dire qui, simultanément, affectent des significations à l'ensemble du dispositif institutionnel et réifient ce système en un modèle "naturel" qui doit être observé sous peine de sanction. On peut, à la lumière de l'histoire institutionnelle du développement, reconstituer le processus d'émergence de la culture des "développeurs" et sa cristallisation, sans grande rupture depuis la période coloniale, en identifiant deux éléments permanents de ce système de normes et de significations.

a) La composante bureaucratique de la culture du Développement

6 Les représentations que les Développeurs se font d'eux-mêmes et de leurs pratiques sociales réfèrent, classiquement, à l'ordre légal-rationnel et bureaucratique caractéristique de la culture occidentale (Weber, 1991) et à "l'artificialisme" découlant du système de valeurs individualiste et positiviste (Dumont, 1985). Le développement rural n'est, à ce niveau, que le transfert de la croyance en l'ingénierie sociale que la culture occidentale s'est d'abord appliquée à elle-même et qu'elle a ensuite mise en œuvre dans les territoires coloniaux

7 On peut en effet constater dans l'histoire coloniale que les responsables de la mise en valeur agricole établissent précocement une analogie entre les paysans africains et les "paysans arriérés" de la métropole, "exploitant leur domaine d'une façon presque aussi primitive qu'en Afrique le paysan noir", mais que "la puissance de la mutualité agricole a transformés en un quart de siècle" (Henry, 1922). Simultanément, les politiques de développement agricole dans les colonies s'inspirent des politiques de modernisation agricole dans les métropoles mais aussi, visiblement, des actions de "travail social" qui s'étendent dans les métropoles à partir du début du siècle (Verdès-Leroux, 1978). Les Développeurs s'identifient à des agents du Progrès organisant le transfert du modèle 
bureaucratique (et, accessoirement, démocratique) pensé comme universel tant au plan de sa légitimité que de son efficacité.

8 L'idéal bureaucratique auquel s'identifie la culture moderne de l'Occident s'efforce de combiner les valeurs d'efficacité et d'équité, et le terrain colonial ne constitue nullement une exception. Le Développement devient une idée-valeur universelle; l'organisation coloniale et ses agents pouvant être identifiés non plus seulement comme le résultat contingent et aléatoire des nécessités du commerce, de la diplomatie ou de l'esprit missionnaire, mais bien comme l'instrument d'une culture rationnelle fondée sur l'idée de l'application organisée d'un savoir pragmatique. Passée la phase proprement militaire et diplomatique de la pénétration coloniale, l'exotisme du terrain africain affecte de moins en moins la nature du savoir-faire et des compétences attendues de l'administrateur, de l'expert ou du technicien colonial, désormais soumis à des normes professionnelles routinisées analogues à celles qui prévalent dans les métropoles. Certes l'exotisme n'est pas entièrement évacué de la "culture coloniale" ; il demeure important, mais davantage comme un élément distinctif valorisant (notamment à l'égard de la "bureaucratie" métropolitaine, le terme prenant ici le sens péjoratif courant) que comme un élément discriminant de l'identité sociale des acteurs. Les Indépendances n'apportent guère de changements en ce qui concerne les "agents expatriés" du développement. Administrateurs et experts coloniaux se coulèrent d'ailleurs sans difficultés dans les institutions d'aide et de coopération ou dans les bureaux d'études, bénéficiant assez souvent de leur attitude antérieure compréhensive à l'égard des revendications nationalistes.

9 En ce qui concerne les nouvelles structures nationales d'administration du développement, la situation est plus complexe et exigerait une analyse qui ne peut être qu'ébauchée ici: a) Un premier point est la continuité formelle de la légitimité bureaucratique dans ce que l'on appelle significativement le "secteur" ou le "monde du développement", qu'il s'agisse d'agences gouvernementales, internationales ou non-gouvernementales ; b) Un deuxième point est la bureaucratisation inéluctable des organisations issues du milieu rural lui-même dès lors que, pour accéder aux ressources $\mathrm{du}$ "secteur du développement", elles sont tenues de répondre aux exigences organisationnelles explicites des bailleurs de fonds (ces exigences n'étant pas moins contraignantes dans le cas des O.N.G.). Plus que jamais la conformité réelle ou apparente aux normes d'efficacité et d'équité du modèle bureaucratique est une condition d'accès au "monde du développement" ; c) Un troisième point, conséquence du précédent, est la croissance en milieu rural d'une couche sociale d'intermédiaires entre le dispositif officiel de développement et les bénéficiaires de son intervention. Ces agents, originaires du milieu rural ou y possédant des relations, valorisent de manière de plus en plus professionnelle leurs connaissances du fonctionnement ou du système de normes des organisations de développement en les mettant au service des organisations ou des associations paysannes.

Dans tous les cas, loin de s'affaiblir avec la crise économique et sociale que traverse actuellement l'Afrique, la composante de la "culture du développement" fondée sur l'idéal-type bureaucratique semble au contraire se renforcer et étendre son influence. L'auto-organisation de la "société civile" que les tenants du "désengagement de l'Etat" appellent de leurs vœux ne semble pas traduire autre chose que la diffusion du modèle "rationnel" d'organisation bureaucratique hors de la sphère étatique, comme ce fut le cas dans les entreprises à partir de la Révolution industrielle en Occident. 
b) La composante populiste de la culture du Développement

11 Ce qui vient d'être dit jusqu'ici de la récurrence des représentations que les développeurs se font d'eux-mêmes et de leurs pratiques sociales évoque le simple transfert de la culture bureaucratique rationnelle occidentale. Les auteurs qui critiquent le processus "d'occidentalisation du monde" s'en tiennent souvent à cet aspect en n'y voyant que la manifestation d'une acculturation dominée par l'idéologie de la modernité occidentale de l'ère industrielle. Un second niveau de la culture du développement doit être combiné à celui-ci. Il concerne cette fois les représentations que les Développeurs se font des "Développés", de leurs pratiques sociales et de leur altérité vis-à-vis du milieu social et culturel occidental.

Nous avons vu qu'aux alentours de la première guerre mondiale est formalisée, du côté britannique comme du côté français, une doctrine du développement rural posant comme incontournable le développement d'un petit paysannat à la fois ancré dans la "coutume", adapté au marché et acquis à l'amélioration de ses conditions de vie.

Ce que A. Phillips (Phillips, 1989) appelle "the West African Policy" ou la théorie du "Paysannat" des experts coloniaux français consiste à défendre contre les effets pervers de l'évolution économique et sociale le "vieux fonds paysan communautaire africain", tel que se le représentent ces experts, et, pour le défendre, l'aider à s'adapter sans perdre ses qualités intrinsèques (communautarisme, solidarité, égalitarisme...).

On sait qu'en réalité - c'est le paradoxe majeur des représentations occidentales sur le développement - l'agriculture africaine, que les experts tiennent régulièrement pour traditionnelle depuis près de quatre-vingts ans, est le produit d'une histoire qui couvre de profondes transformations du tissu politique et social africain depuis notamment le début du XIX ${ }^{e}$ siècle. Loin de représenter le "fond paysan africain ancien" qu'il faudrait préserver des effets délétères du capitalisme sauvage et des "élites évoluées", les petites exploitations africaines, produisant ou non pour l'exportation, sont au contraire le produit d'une histoire sociale éminemment mouvante et contemporaine. Cette image idéalisée des agriculteurs ouest-africains et la politique coloniale qu'elle inspire relèvent du populisme, c'est-à-dire d'un certain type de rapport social (idéologique, moral, scientifique, politique) que des intellectuels nouent au moins symboliquement avec le "peuple" à partir de la représentation qu'ils s'en font (Olivier de Sardan, 1990 ; Passeron, 1989). Le "populisme colonial" se rattache, sans aucun doute possible, à la configuration idéologique du populisme qui, sous des formes qui peuvent être très différentes voire opposées, s'élabore en Occident à partir de la fin du XIX siècle. L'affinité profonde entre l'idéologie populiste et le Développement a été évidemment soulignée par différents auteurs mais, le plus souvent, en se rapportant à la période postérieure à la seconde guerre mondiale. La filiation est en réalité plus ancienne et s'instaura d'emblée dès que les colonisateurs éprouvèrent le besoin de se doter d'un savoir qui se voulait pragmatique.

On peut conclure sur ce point que la "construction sociale du réel africain" par les Développeurs est beaucoup plus complexe que le simple transfert de l'idée évolutionniste $d u$ Progrès universalisé: la culture du développement charrie simultanément la nostalgie pour les communautés "naturelles", endémique dans la culture occidentale (Robertson, 1984). L'image communautaire est toujours restée très forte dans les conceptions et la mise en pratique des projets de développement, et régulièrement réactualisée par des constructions théoriques peu attentives à l'histoire rurale africaine (par exemple, récemment, Hyden et sa notion "d'économie affective", 
ou, de manière plus nuancée, la thèse de la "revanche des sociétés africaines" du politologue J.-P. Bayart).

C'est dans la combinaison contradictoire de cette représentation populiste des Développés, d'un côté, et de la valorisation du modèle d'action rationnel et bureaucratique des Développeurs, de l'autre, qu'il faut rechercher la raison de la prégnance et de la récurrence du modèle participatif de développement comme figure paradigmatique de la culture du développement.

Le populisme "bureaucratique" et sa reproduction : la procédure de déplacement des contradictions

On peut en effet montrer, en développant les arguments présentés dans notre article précédent : a) que la combinaison des valeurs bureaucratique et communautaire génère des contradictions; b) que ces contradictions sont perçues par la culture du développement comme le résultat "d'effets pervers" indépendants de la validité du modèle populiste et bureaucratique de connaissance et d'action ;

18 a) Nous appellerons "populisme bureaucratique" l'idéal-type de l'autorité et de la légitimité caractéristique des valeurs de la culture du Développement. Il prétend en définitive concilier les vertus de l'efficacité dépersonnalisée de la Bureaucratie (entendue comme idéal-type d'organisation) et les vertus de la Communauté, de la Solidarité et des particularismes qui sont censés régir les paysanneries africaines. Dans ce dispositif de connaissance et d'action, le populisme légitime en outre le statut et l'action des opérateurs de développement.

19 De nombreuses études ont toutefois décrit comment la combinaison de ces éléments contradictoires que sont l'idéal bureaucratique et l'idéal communautaire aboutit simultanément à un renforcement du formalisme bureaucratique d'une part, et, d'autre part, au renforcement de micro-réseaux particularistes assimilé à l'expansion du clientélisme et de la corruption. D'un côté, les dysfonctionnements des structures locales de développement sont attribués au particularisme des sociétés et cultures locales, et corrigées par davantage de règles et de contrôle; d'un autre côté, les solutions pragmatiques pour adapter ces règles sont considérées comme manifestation d'inaptitude et de corruption; néanmoins les caractéristiques communautaires des collectivités paysannes sont sollicitées pour assurer aux organisations paysannes cohérence, représentativité et durée. A partir de là, se déroule un processus de "malentendu productif" et d'ajustement sans fin entre les "stratégies paysannes" (révélant bien évidemment l'hétérogénéité sociale et économique du milieu paysan) et les stratégies des opérateurs de développement (révélant le caractère politique et contraignant de leur action ainsi que l'existence de pratiques très éloignées des règles formelles de l'organisation rationnelle). La lutte contre les dysfonctionnements du "populisme bureaucratique" alimente la croissance de la bureaucratie en même temps qu'elle nourrit les stratégies paysannes de "détournement" des objectifs bureaucratiques.

20 b) Les administrateurs, experts et techniciens coloniaux n'étaient pas moins au fait de ces "effets inintentionnels" que les critiques contemporains du style technocratique de développement. La culture du développement s'est ainsi cristallisée comme un système cognitif confronté en permanence à des effets non attendus ou contre-productifs qui entravent son instrumentalisation. Il s'en suit que, beaucoup plus que sur une codification de critères professionnels spécifiques à "l'action pratique de développement" en soi, la culture du développement s'est professionnalisée 
principalement sur la base d'une codification des effets, invariablement perçus comme pervers, qui s'opposent à cette action.

On peut observer, en premier lieu, que si les différentes "spécialités" du développement possèdent bien un corpus de normes techniques, celui-ci concerne des codes professionnels spécialisés qui permettent aux agronomes ou aux zootechniciens ou aux sociologues ou aux économistes etc., de se situer et de communiquer au sein de leurs spécialités, mais généralement pas entre eux. L'intégration de ces véritables "corps spécialisés" est plutôt assurée par une hiérarchisation implicite des domaines de compétence, ou par un ordre d'intervention des spécialités dans le processus de l'intervention ou encore par une prise en charge de la part des spécialités dominantes des aspects les plus généraux du développement (par exemple les aspects sociaux, voire culturels, par les économistes et les agronomes) (Schmitz, 1991). Autrement dit, il n'existe pas de normes professionnelles générales propres à l'activité de "développer" en tant que telle, si ce n'est celles qui émanent des spécialités dominantes et qui, par là-même, sont susceptibles d'être toujours remises en question. L'évaluation des résultats d'une action de développement relève d'ailleurs d'une alchimie notoire (où l'on retrouve la prééminence de l'économie) et il n'y a pas de consensus absolu sur ses techniques (comme le manifeste, par exemple, la lutte d'influence que se livrent les grandes agences de développement pour imposer leur propre système d'évaluation).

On peut remarquer, ensuite (surtout en ce qui concerne les groupes d'agents appartenant aux niveaux les plus élevés des structures de développement), qu'il n'existe guère de "sanction du réel" pour les professionnels du développement. Les échecs (ou les réussites, plus rares) des projets ou des politiques économiques ne se traduisent pas vraiment par des mesures professionnelles vis-à-vis des opérateurs de développement s'ils satisfont par ailleurs aux normes de leur spécialité. D'ailleurs, il existe des raisons "objectives" pour que ce poisson-là reste définitivement noyé : d'une part, comme on vient de le signaler, la difficulté d'évaluer les effets propres d'une intervention à partir de critères simples, univoques et reconnus; d'autre part, le fait qu'une action de développement met en œuvre une telle multiplicité d'agents et de structures qu'il est illusoire de vouloir en imputer individuellement les résultats. Le système d'évaluation du savoir-faire professionnel des Développeurs repose donc surtout sur des normes strictement internes aux organisation de développement, et elles sont en général d'ordre politique : un chef de service de la Coopération ou un chef de projet n'est pas évalué sur la réussite des opérations qu'il a montées ou dirigées mais plutôt sur l'engagement de la masse de crédits prévue en fonction de directives politiques (Freud, 1991) ou sur la qualité de la coordination avec les responsables politiques et administratifs des pays bénéficiaires (Le Roy, 1991).

23 Si l'on admet que la fonction d'un système de nonnes est de réduire l'incertitude dans l'accomplissement des activités sociales, ni les nonnes techniques "de spécialités" ou de disciplines, ni les normes internes aux organisations ne sont donc en mesure de fournir un tel système normatif face à l'incertitude des résultats de l'action de développement et à la difficulté d'évaluer cette action. Tout se passe comme si le "travail" de la culture du développement consistait à détacher cette incertitude et cette difficulté de l'action-même de "développer", et à l'imputer à des effets récurrents indépendants du modèle du Populisme bureaucratique lui-même.

Dans ces conditions, l'on conçoit que les efforts de correction de ces effets pervers par le dispositif populiste-bureaucratique conduisent simultanément à la reproduction sans 
fin des contradictions et au renforcement de ce dispositif dès lors que sa légitimité n'est pas remise en cause. L'exercice spécifique du développement a surtout consisté à penser et à codifier ces éléments étranges et inattendus qui accompagnent l'action de "développer", et dont l'existence atteste a contrario l'objectivité de la culture du développement. Tout se passe en effet comme si, plus les obstacles au développement étaient importants et variés, plus ils fondaient "en raison" l'exigence du développement, plus était conforté le savoir qui l'objective et plus était légitime l'autorité qui le promeut. Quels que soient leurs champs de compétence, leurs idéologies politiques ou leurs organisations de rattachement, les Développeurs ne peuvent qu'être d'accord pour agir pour le développement - car on ne peut être contre le Progrès - et pour admettre que l'action de développement devra toujours composer avec la double nécessité d'une organisation bureaucratique efficace, impliquant l'identification d'interlocuteurs privilégiés en milieu paysan, et d'une participation de la base des "bénéficiaires", impliquant la mobilisation des ressources communautaires.

Le populisme bureaucratique et ses variations : les procédures de réactualisation de la culture du développement

La procédure de "déplacement des contradictions" qui vient d'être décrite explique sans doute qu'une fois "inventée" l'image populiste de la paysannerie africaine, l'écart entre les effets attendus de l'intervention rationnelle de développement et les résultats observables puisse être interprété sans que soit remise en cause le système de valeurs du populisme bureaucratique. Néanmoins il reste à expliquer la permanence de la "carte cognitive" de la culture du développement elle-même, et notamment la manière dont la fonction critique, inhérente à la bureaucratie rationnelle, non seulement épargne mais encore conforte les idées-valeurs de la culture du développement. Quelles sont les structures conceptuelles récurrentes qui, tout au long de l'histoire institutionnelle du développement rural, ont organisé la connaissance et la construction de l'expérience des agents du développement?

Il faut revenir sur le statut central des "effets pervers" dans les procédures d'information et de prise de décision de ces agents. Ces effets pervers sont en effet simultanément rejetés dans la pure contingence mais sans cesse ramenés à la conscience des développeurs par le jeu de la fonction critique inhérente à l'exercice de la rationalité bureaucratique. L'écart, imputé à ces effets pervers contingents, entre les objectifs rationnels du développement et les résultats atteints est invariablement expliqué par trois types fondamentaux de considérations: a) soit par les défauts de l'organisation bureaucratique elle-même lorsqu'elle se transforme en une technocratie ; b) soit par l'intervention perverse d'intérêts émanant de couches sociales dominantes non-paysannes; c) soit encore par l'état de décomposition du milieu paysan lui-même qui, sous l'effet de la domination exogène, perd ses qualités spécifiquement "populaires" et se trouve gagné par l'individualisme, l'inégalité ou tout simplement l'anomie engendrée par une grande détresse matérielle et morale.

Ces arguments relèvent de deux variantes ou registres (Olivier de Sardan, 1990) du populisme que l'on peut, à la suite de Passeron (Grignon et Passeron, 1989), définir de la manière qui suit. Le premier registre, ou populisme proprement dit, fait retour au "Peuple" en lui attribuant des qualités spécifiques et une autonomie réelle. C'est l'argumentation fondée sur les savoir paysans, la cohérence du mode de vie communautaire et le développement "à la base" à partir des besoins tels que les ressentent les paysans eux-mêmes. Les effets pervers sont alors imputés aux deux 
premiers types d'argumentaire ( $a$ et $b$ ) précédemment indiqués: perversion de l'organisation bureaucratique, lorsqu'elle se transforme en une technocratie coupée des administrés, ou intervention d'intérêts émanant de couches sociales dominantes non-paysannes (politiciens, administrateurs, commerçants, intérêts économiques et politiques étrangers), lorsque cette intervention empêche que s'expriment les besoins authentiques et que se réalisent les initiatives paysannes. En termes d'action, le populisme proprement dit postule que seul le "Peuple" est en mesure de trouver les solutions à ses propres problèmes et qu'il en possède les ressources suffisantes. Le populisme stricto sensu surestime la composante communautaire et populaire comme élément régulateur du modèle populiste-bureaucratique.

Le second registre est celui du misérabilisme ou du "dominocentrisme", qui reconnaît aussi la nécessité de fonder la connaissance et l'action sur la réalité populaire mais qui se polarise sur les effets non seulement pervers mais encore destructeurs qu'exercent les couches sociales dominantes sur le "Peuple". Les processus de domination tendent alors à faire disparaître les ressources spécifiques et la marge d'autonomie des sociétés et des cultures populaires C'est le troisième type d'argumentaire (c). En termes d'action, cela signifie que la paysannerie n'est pas en mesure de générer et de mettre en œuvre par elle-même les solutions à ses problèmes et que c'est le rôle du dispositif de développement de créer les conditions favorables à la réhabilitation des capacités d'initiatives locales. Le misérabilisme conduit à surestimer la composante bureaucratique-rationnelle comme élément régulateur $\mathrm{du}$ modèle populiste-bureaucratique.

Toutefois, ainsi que l'a montré Olivier de Sardan, populisme et misérabilisme procèdent d'une même matrice: le populisme moral, et se situent dans un même registre expressif : celui du stéréotype (Olivier de Sardan, 1990). Les variantes populiste et misérabiliste, loin de s'opposer, peuvent facilement permuter ou se compléter au sein de la "culture du développement" sans contradiction apparente. On retrouve d'ailleurs l'argumentaire populiste et l'argumentaire misérabiliste combinés en proportions variables aussi bien au sein des théories libérales du développement qu'au sein des théories de la dépendance ou encore chez un même auteur (par exemple Cham bers : Olivier de Sardan, 1990). Cela explique également que, dans le processus historique de constitution de la culture du développement, le modèle du développement participatif est relativement indépendant des pratiques dans la sphère proprement politique (Chauveau, 1992).

30 Mais le point important pour notre propos est que la combinaison du populisme et $\mathrm{du}$ misérabilisme, leur coexistence ou leur opposition dans une même configuration cognitive et praxéologique suscitent, par une sorte de balancement d'un registre à l'autre, la redécouverte constante du problème de la Participation populaire et le réaménagement incessant des composantes populistes et bureaucratiques au sein du modèle populiste bureaucratique. Tout se passe comme si la variabilité de la combinaison entre populisme et misérabilisme, provoquée en permanence par le jeu de la fonction critique au sein de la composante bureaucratique, réactivait du même coup l'ensemble des valeurs du populisme bureaucratique et suscitait la "réinvention" constante et régulière $\mathrm{du}$ développement participatif comme "prototype" $\mathrm{du}$ développement.

31 Ce faisant, le dispositif d'intervention réamorce la production d'effets d'ajustement des rapports sociaux entre Développeurs et Développés, entre les différentes couches 
rurales et entre les diverses composantes organisationnelles et hiérarchiques du dispositif de développement lui-même. Ce sont ces processus d'ajustement socialement "normaux" - que la culture du développement engendre et codifie comme des processus pervers indépendants de, ou ne remettant pas en cause la légitimité du Populisme bureaucratique, ménageant ainsi les conditions d'un nouveau cycle d'ajustement. Loin de déstabiliser la reproduction du populisme bureaucratique et de révéler ses propres effets pervers, toute variation dans la combinaison des composantes bureaucratique et populiste, ou des registres populiste et misérabiliste a pour effet de relancer la quête de la Participation paysanne.

On peut identifier dans l'histoire institutionnelle du développement quelques sources typiques de variabilité qui renforcent la capacité de résilience du populisme bureaucratique (on pourrait presque parler d'un système cognitif ou de valeurs "homéostatiques"). C'est ici - mais, nous semble-t-il, ici seulement - qu'il est nécessaire de réintroduire les "intérêts" des acteurs et des groupes d'acteurs. C'est en effet dans le cadre cognitif et normé du populisme bureaucratique que les acteurs se construisent leurs intérêts et élaborent les stratégies pour les promouvoir.

On peut penser par exemple, parmi les sources possibles de variation, aux effets des conjonctures historiques. Par exemple, les périodes de crises ressenties à l'échelle internationale semblent d'abord accentuer le registre misérabiliste, donc la composante bureaucratique de la culture du Développement (on tente d'organiser les populations frappées par la crise), puis, dans un second temps, recourt au registre populiste proprement dit (on en appelle à l'auto-organisation des populations), appelant la réactualisation du modèle participatif par le renforcement de la composante populiste. Ce fut le cas en Afrique de l'Ouest durant la crise des années 1930, à la fin de la seconde guerre mondiale et depuis la crise des années 1970. Il faut souligner que ces périodes correspondent aussi à un désengagement forcé de l'Etat ou des bailleurs de fonds, et il n'est pas impossible que la redécouverte des capacités d'organisation et d'initiatives paysannes (ou de la "société civile") soit une manière, pour les opérateurs de développement, de faire de nécessité vertu...

Un autre facteur de variabilité de la combinaison populisme/misérabilisme (et donc de réactualisation du débat sur la Participation populaire) est la position des agents dans l'organisation hiérarchique des structures de développement. Il est probable qu'en privilégiant comme nous l'avons fait ici les discours et les pratiques des instances ou acteurs prééminents dans le "monde du développement", nous surestimons en partie le registre populiste et la composante communautariste de la culture du développement. En effet, les agents subalternes du dispositif de développement, plus directement impliqués dans les relations locales de pouvoir, semblent plus sensibles au registre misérabiliste et à la composante bureaucratique du modèle participatif (comme semble l'indiquer le ton proche du paternalisme autoritaire utilisé par les techniciens ou les agents "de base" dans leurs confidences "off the record"). Ici, ce sont les contradictions d'intérêts entre les différentes catégories d'opérateurs de développement qui peuvent générer une différence de sensibilité à l'égard des valeurs participatives et, en fonction des caractéristiques et de la situation de l'organisation concernée, réactiver dans un sens bureaucratique/misérabiliste ou populiste l'enjeu participatif.

On peut aussi envisager comme facteur de variabilité les particularités nationales ou la "culture d'organisation" des grandes agences de développement. A l'époque coloniale, l'administration française semble ainsi avoir davantage cultivé le misérabilisme que le 
populisme, à l'inverse des Britanniques (l'Indirect Rule traduisant ce penchant). Aujourd'hui, il est frappant de constater que la rivalité des principaux bailleurs de fonds s'exprime notamment à propos de l'organisation de la vulgarisation agricole et de l'encadrement des agriculteurs (Training and Visit, Recherche/Développement, Conseil de Gestion...). En y regardant de plus près, on constate peu de différences entre ces doctrines si ce n'est, ici encore, l'accent mis, à un moment donné, sur l'une des composantes du populisme bureaucratique ou sur l'un des registres du populisme. Les agences de développement peuvent d'ailleurs nuancer leurs doctrines selon les conjonctures et défendre les mêmes opinions que leurs rivales à des moments différents...

Autant de "mini-variations" qui entretiennent les débats sur le "prototype participatif', occulte la répétitivité de l'argumentaire et protège de toute remise en cause les schémas conceptuels de la culture du développement.

\section{BIBLIOGRAPHIE}

Baré, J.-F., 1987 - "Pour une anthropologie du développement économique", Études Rurales, 105-106: 267-298.

Bastide, R., 1971 - Anthropologie appliquée, Paris, Payot.

Chauveau, J.-P., 1992 - "Le "modèle participatif' est-il alternatif?", Bulletin de l'APAD, 3: 20-30.

Dumont, L., 1977 - Homo aequalis, Paris, Gallimard.

Dumont, L., 1985 - "Identités collectives et idéologie universaliste: leur interaction de fait", Critique, XLI, 456: 506-518.

Foucault, M., 1966 - Les mots et les choses, Paris, Gallimard.

Freud, C., 1991 - "Qu'attendent les bailleurs de fonds des anthropologues?", Bulletin de l'APAD, $1: 22$.

"Grignon, C., Passeron, J.-C., 1990 - Le savant et le populaire. Misérabilisme et populisme en sociologie et en littérature, Paris, Gallimard et Le Seuil.

Henry, Y., 1922 - Le programme agricole, Paris, Larose.

Kitching, G., 1982 - Development and Underdevelopment in historical perspective. Populism, nationalism and industrialization, London and New York, Methuen.

Le Roy, E., 1991 - "L'expertise internationale en Afrique: le cas de l'expertise juridique sur les questions foncières", Bulletin de l'APAD, 2:16-25.

Olivier de Sardan, J-P., 1990 - "Populisme développementiste et populisme en sciences sociales: idéologie, action, connaissance", Cahiers d'Etudes Africaines, 120: 475-492.

Phillips, A., 1989 - The Enigma of Colonialism. British Policy in West Africa, London, James Curry.

Richards, P., 1985 - Indigenous agricultural revolution, London, Hutchinson. 
Robertson, A.F., 1984 - People and the State. An anthropology of planned development, Cambridge University Press.

Schmitz, J., 1991 - "Les sciences et techniques appliquées au développement et l'anthropologie", Bulletin de l'APAD, 2: 9-15.

Verdès-Leroux, J. 1978 - Le travail social, Paris, Éditions de Minuit.

Weber, M., 1991 - Histoire économique. Esquisse d'une histoire universelle de l'économie et de la société, Paris, Gallimard.

\section{AUTEUR}

\section{JEAN-PIERRE CHAUVEAU}

ORSTOM - Institut Français de Recherches pour le Développement en Coopération 